\title{
Stardust in meteorites: A link between stars and the Solar System
}

\author{
Ernst Zinner \\ Laboratory for Space Sciences and the Physics Department, Washington University, St. Louis, \\ MO USA \\ email: ekz@wustl.edu
}

\begin{abstract}
Ultimately, all of the solids in the Solar System, including ourselves, consist of elements that were made in stars by stellar nucelosynthesis. However, most of the material from many different stellar sources that went into the making of the Solar System was thoroughly mixed, obliterating any information about its origin. An exception are tiny grains of preserved stardust found in primitive meteorites, micrometeorites, and interplanetary dust particles. These $\mu \mathrm{m}$ - and sub- $\mu \mathrm{m}$-sized presolar grains are recognized as stardust by their isotopic compositions, which are completely different from those of the Solar System. They condensed in outflows from late-type stars and in SN ejecta and were included in meteorites, from which they can be isolated and studied for their isotopic compositions in the laboratory. Thus these grains constitute a link between us and our stellar ancestors. They provide new information on stellar evolution, nucleosynthesis, mixing processes in asymptotic giant branch (AGB) stars and supernovae, and galactic chemical evolution. Red giants, AGB stars, Type II supernovae, and possibly novae have been identified as stellar sources of the grains. Stardust phases identified so far include silicates, oxides such as corundum, spinel, and hibonite, graphite, silicon carbide, silicon nitride, titanium carbide, and Fe-Ni metal.
\end{abstract}

\section{Discussion}

HenNing: Is there any hope to do the same work to iron sulphide grains? As you know, it's strongly debated whether iron sulphide is produced in the protoplanetary disk or in the molecular cloud.

ZINNER: Well, one question is of course how stable sulphide grains are. One type of non-solar grain has been found because it could be extracted from meteorites and then studied, and the other type is actually studied in situ. The only hope is to find some sulphide grains in situ and then measure their sulphur isotopic compositions. This is not too hard to do in principle, but not much has been done yet. It's still possible that they exist.

Cruikshank: Scott Sandford tells me that sulphides are well represented in the Stardust samples. Is that still true?

ZinNeR: There is also in a lot of iron sulphide in meteorites, but most of it is not of stellar origin. So it's the same as the silicate grains. The Solar System makes silicate grains and so you have to measure thousands of them in order to find the stellar ones. The same thing might be necessary for the sulphide grains, but not much has been done.

ZIURYS: What do you expect for the magnesium isotope ratio between AGB stars and supernova? 
ZINNER: There are differences, but it's a little complicated. There are always large anomalies in ${ }^{26} \mathrm{Mg}$, but this is from the decay of ${ }^{26} \mathrm{Al}$, so you cannot say anything about the $\mathrm{Mg}$ itself. You have to examine the ${ }^{25} \mathrm{Mg}:{ }^{24} \mathrm{Mg}$ ratio. There are very large variations that we think are from AGB stars because oxygen isotopes in the same grains suggest they are from AGB stars. There are a few grains which we think are of supernova origin, and $\mathrm{Mg}$ is also anomalous in 25:24 in them. Making these measurements also depend on how much $\mathrm{Mg}$ is in the grains. $\mathrm{Mg}$ is an element that doesn't like to go into silicon carbide. However, $\mathrm{Al}$ goes there and then you find almost mono-isotopic ${ }^{26} \mathrm{Mg}$ from the decay of ${ }^{26} \mathrm{Al}$. $\mathrm{Mg}$ is more volatile and doesn't like to go into silicon carbide or graphite, so it's much more difficult to do such measurements.

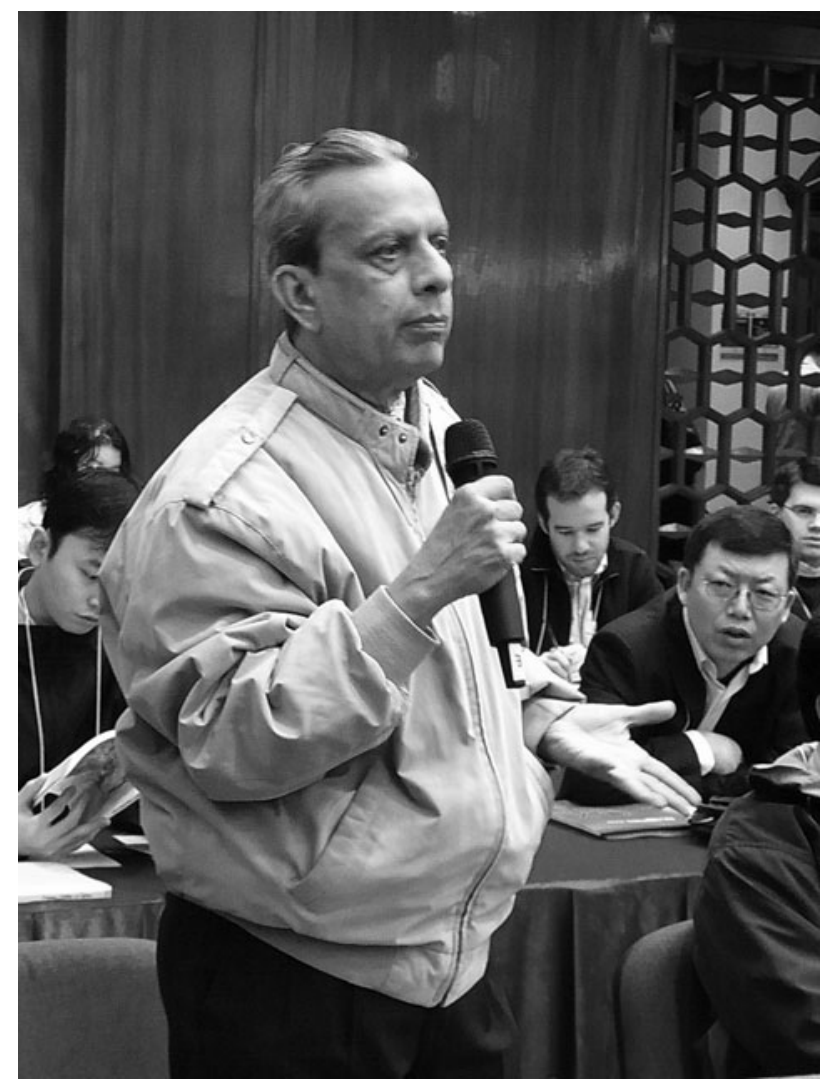

D.B. Vaidya asking a question. 